\title{
European Guidelines on the Organisation of Breast Centres and Voluntary Certification Processes
}

\author{
Laura Biganzolia $^{\mathrm{a}}$ Lorenza Marotti $^{\mathrm{b}}$ Maria-Joao Cardoso ${ }^{c}$ Luigi Cataliotti $^{\mathrm{d}}$ \\ Giuseppe Curigliano $^{e, f}$ Jack Cuzick ${ }^{g}$ Aaron Goldhirsch $^{\text {h, }}$ Marjut Leidenius $^{j}$ \\ Robert Manselk $^{k}$ Christos Markopoulos' Lynda Wyldm Isabel T. Rubion,o \\ ${ }^{a}$ Breast Unit, Nuovo Ospedale di Prato, Prato, Italy; ${ }^{b}$ EUSOMA, Florence, Italy; ${ }^{\mathrm{c} B r e a s t}$ Unit, Champalimaud Clinical

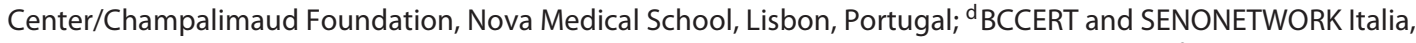 \\ Florence, Italy; ${ }^{\mathrm{e}}$ Department of Oncology and Haematology, University of Milan, Milan, Italy; ${ }^{f}$ IEO, European \\ Institute of Oncology IRCCS, Milan, Italy; ${ }^{9}$ Wolfson Institute of Preventive Medicine, Queen Mary University of \\ London, London, UK; ${ }^{\mathrm{h}}$ International Breast Cancer Study Group, Bern, Switzerland; ${ }^{\mathrm{i} M u l t i M e d i c a, ~ M i l a n, ~ I t a l y ; ~}{ }^{\mathrm{j} B r e a s t}$

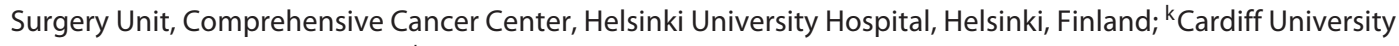 \\ School of Medicine, Cardiff, UK; 'Medical School, National and Kapodistrian University of Athens, Athens, Greece; \\ m Department of Oncology and Metabolism, University of Sheffield, Doncaster and Bassetlaw Teaching Hospitals \\ NHS Foundation Trust, Doncaster, UK; " Breast Surgical Oncology Unit, Clinica Universidad de Navarra, Madrid, \\ Spain; `Universidad de Navarra, Pamplona, Spain
}

\section{Keywords}

Multidisciplinary · Quality control · Breast centre · Certification · Quality indicators

\begin{abstract}
Background: EUSOMA undertook the commitment of defining the requirements for a specialist breast centre, which has become the reference document for the implementation of breast centres. Summary: The EUSOMA requirements for a specialist breast centre give clear indications regarding the requisite caseload, dedicated team composition (core and non-core team), organisation, availability of services and equipment throughout the patient pathway, quality control, and application of a multidisciplinary approach. The minimum number of cases is 150 newly diagnosed breast cancer cases per year. Based on the EUSOMA requirements, a voluntary and accredited certification scheme has been developed. In Europe, other voluntary certification schemes are available, such as those developed by the German Cancer Society and German Society for Breast Disease, the National
\end{abstract}

Cancer Peer Review Programme in the UK, and the "label de qualité" established by the Swiss Anticancer League and the Swiss Senology Society. The European Commission Initiative on Breast Cancer (ECIBC) has overseen the development of a European Quality Assurance Scheme. Key Messages: Nearly 20 years after the initial publication of the EUSOMA requirements, ensuring that all breast cancer patients in Europe are treated only in certified breast centres should be considered a high priority and eventually achieved through collaborative efforts.

(c) 2019 S. Karger AG, Basel

\section{Introduction}

In Florence in 1998, the first European Breast Cancer Conference (EBCC-1) took place, jointly organised by EUSOMA, EORTC, and EUROPADONNA. The "manifesto," voted on and ratified by nearly 1,000 participants in the conference - the so called "Florence Statement" set an agenda on key issues in breast cancer research,

\section{KARGER}

(C) 2019 S. Karger AG, Basel 
treatment, prevention, and advocacy [1], and presented a challenge for EUSOMA to establish the definition of requirements for specialist breast centres. The subsequent publication of the EUSOMA requirements [2] opened the way to a growing commitment, both from the scientific community and advocates to uphold the re-organisation of breast cancer care in Europe through the implementation of dedicated centres. Health professionals involved in the treatment of breast cancer generally welcomed these changes with great enthusiasm, and committed to the required re-organisation of their daily work, on the basis of the concept of multidisciplinary and dedicated patient pathways leading to better outcomes for patients.

However, it cannot yet be stated that all breast cancer patients across Europe have access to fully equipped multidisciplinary and multiprofessional breast centres [1]. Due to the combined efforts of health professionals, advocates, and policy makers, in 2003 and 2006 two parliamentary resolutions on breast cancer were published [3, 4]. The first resolution called for all patients with breast cancer to be entitled to be treated by a dedicated multidisciplinary team, and the second to ensure nationwide provision of interdisciplinary breast units in accordance with the EU guidelines by 2016. This was deemed necessary as treatment within an interdisciplinary breast unit has been shown to improve the chances of survival and quality of life. Despite this, and other initiatives developed during the ensuing years [5], the 2016 EBCC manifesto on breast centres identified that the 2016 deadline for all patients in EU countries to access breast centres was not be met by most countries. As such, the manifesto subsequently called on policy makers and politicians to ensure that patients with breast cancer in Europe are treated in a specialist breast centre, as soon as possible [6].

\section{Breast Centres}

The EUSOMA recommendations on a specialist breast centre [7] is the reference document for the implementation and effective functioning of a breast centre, for dedicated breast specialists, hospital management, and policy makers. The document gives clear indications on caseload, dedicated team, organisation, availability of services and equipment throughout the patient pathway, quality control, and multidisciplinary approach. The number of referrals to be considered for the establishment of a breast centre is the yearly volume of new breast cancer cases. Each breast centre must treat a minimum of 150 new cases per year, underpinned by evidence suggesting that patient volumes seen in breast centres have an effect on survival $[8,9]$. Similarly, a multidisciplinary approach to care, based on shared patient management by all the necessary specialists, who together decide on the manage- ment plan to be recommended to each patient, has been shown to have a positive effect on survival $[10,11]$.

Each breast centre is required to nominate a clinical lead who is responsible for maintaining issues such as an ongoing multidisciplinary approach to disease diagnosis and management, ensuring an appropriate level of training of team members, and fostering research activity and quality control. The breast centre must integrate multidisciplinary meetings (MDMs), ensuring that each patient with early disease is discussed pre- and post-operatively. Additionally, patients should be discussed throughout the different phases of their disease (at early, recurrent, and/or advanced stages). Multidisciplinary discussion and consensus may result in critical changes to treatment recommendations, ultimately improving upon plans initially conceived without the benefit of multidisciplinary input [12] and may also assist in avoiding unnecessary treatments [13]. All decisions taken during the MDM must be formally recorded. MDMs also play an important role in the training of specialists and facilitates the identification of patients eligible for clinical trials.

With regard to the breast centre team, EUSOMA identifies "core" and "non-core" team members. Core team members are defined as those that are essentially involved in the diagnosis and management of all breast cancer patients. These professionals include the radiologist, radiographer, breast surgeon, pathologist, medical oncologist, radiation oncologist, breast care nurse, and data manager. All other professionals not centrally involved in all cases ("noncore" team members), such as reconstructive surgeons, geneticists, psychologists and nuclear medicine physicians, work collaboratively with the breast centre, joining the MDM when necessary in order to guarantee optimum care.

Each breast centre must also have access to all the necessary infrastructure needed to carry out diagnostic and treatment procedures. Detailed written information (leaflets) on the centre, describing diagnostic and treatment options, should be available at the breast centre, together with information on local support groups and advocacy organisations. The centres must also provide training for junior staff or students at a local and/or an international level. Research represents a very important aspect of the activity of a breast centre and comprises an essential part of training specialists. Clinical trial activity represents a fundamental requirement of a breast centre, with the expectation that the team actively facilitate the participation of patients in trials as appropriate.

Quality control represents a pivotal aspect of the existence of a breast centre. Data collection represents one factor essential to carrying out quality control, as it allows an objective means of checking adherence to guidelines and allows evaluation of the centre's activity against predefined performance and outcome quality indicators. As such, it is mandatory for each breast centre to maintain a 


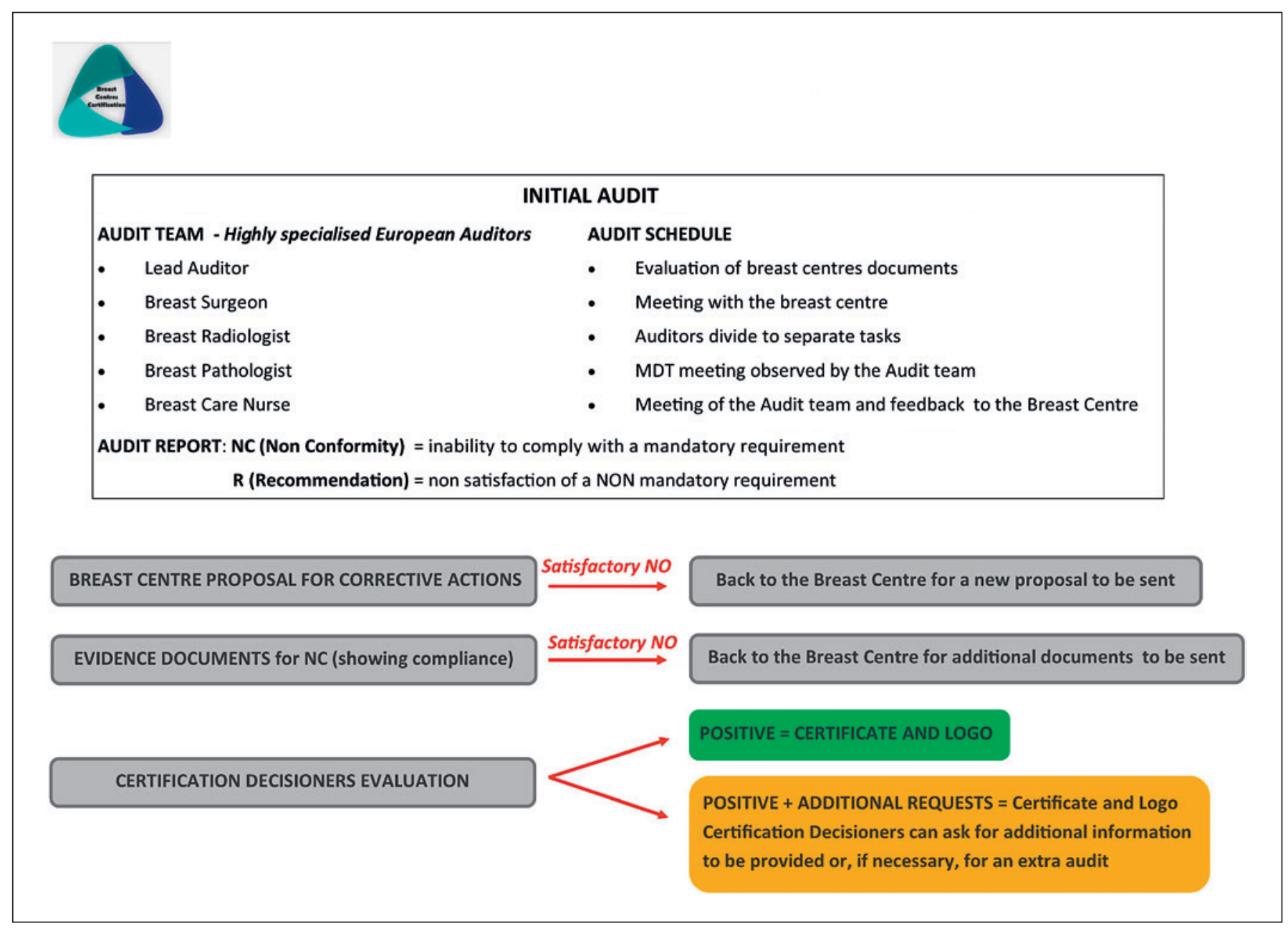

Fig. 1. Certification procedure.

database which collects data about the patients treated in the centre. A data manager, who is responsible for data collection and analysis under the supervision of a designated core team clinician, is also considered essential. Data collection and analysis is fundamental to perform internal audits, update protocols and guidelines, validate quality indicators, and monitor changes in treatment. The breast centre must undertake a formal performance and clinical protocol review meeting to evaluate the breast centre data at least once a year, discussing any related issues, and if necessary, updating protocols.

EUSOMA has issued a set of quality indicators on breast cancer care, which correlate to the different points of the patient pathway from diagnosis to loco-regional treatment, systemic therapy, and follow-up [14]. Sharing a defined set of quality indicators allows breast centres at a local, national, and international level to participate in external benchmarking activities, such as the comparison of one breast centre's results with those of others. This allows both for critical service gaps in care provision to be identified, and also to foster the replication of successful approaches from other centres into others. External audit via a certification process is an effective tool to enact an independent and objective evaluation of defined requirements that will be measured.

Based on the EUSOMA requirements, a voluntary and accredited certification process has been developed. It is managed jointly by the Italcert Conformity Assessment Body and BCCert, a not-for-profit scientific society [15].

The EUSOMA certification process runs on a 3 -year cycle. Entry requirements mandate that the applying breast centre has an established clinical lead, treats at least 150 new cases per year, and uses a database validated by EUSOMA.

EUSOMA has created a data warehouse of prospectively collected information (anonymised patient data). For each breast centre undergoing the certification process, the EUSOMA data centre first validates the breast centre's database in terms of technical aspects, completeness, and accuracy of data collection. Subsequently, the breast centres make a yearly data transfer to the EUSOMA data warehouse. The data centre issues an annual re- 
port for each breast centre under certification, demonstrating the centre's compliance with EUSOMA quality indicators. The updating of the dataset goes along with the updating of the existing QIs and with the introduction of a new set of indicators.

All EUSOMA-certified centres will be part of a network, aiming to develop co-operative scientific projects utilising data provided by the breast centres, which result in publications on specific topics [16]. Regular monitoring, auditing, and benchmarking assist breast centres in maintaining a high standard of care, resulting in better performance of quality indicators [17].

The certification process enables breast centres to improve their quality standards in terms of necessary changes in clinical approaches, and allows objective, constant monitoring, and analysis of centre organisation, activity, and performance. The EUSOMA certification process comprises offsite and on-site activity (Fig. 1). Each year, the breast centre is required to complete a questionnaire which collects detailed information on EUSOMA's requirements, and must transfer data to the EUSOMA data warehouse to allow external assessment of quality indicators. An on-site audit visit to each breast centre is carried out annually. The first year's visit comprises of a day and a half, with each subsequent visit lasting 1 day. The audit team is made up of a group of $5 \mathrm{ex}-$ perts: a lead auditor, radiologist, pathologist, surgeon, and breast care nurse. The audit team meets local colleagues from the different disciplines and physically inspect the breast centre on-site. Going through the patient pathway, the audit team objectively cross-checks compliance with the EUSOMA requirements, both by interacting directly with the breast centre team, and via analysis the internal documents supplied by the centre at the request of the audit team. During the audit the visiting team observes an MDM. At the completion of the visit, the audit team conducts a private internal meeting to prepare the report in which any non-conformity with stated requirements, recommendations, and observations are highlighted. Following the receipt of feedback, the breast centre is required to provide a proposal of corrective actions, which, once accepted by the audit team, must be implemented within 3 months for issues related to non-conformities. If recommendations are made by the audit team, evidence of subsequent actioning will be checked during the next surveillance audit. Once a breast centre has provided documented evidence of a solution to any previously identified non-conformity, this must be evaluated as satisfactory by the audit team. Subsequent to that, the whole evaluation of the specific breast centre will be revised by the "Decisioner Committee." Following positive evaluation, the breast centre will be granted certification. During the ensuing 2 years, a surveillance audit is carried out following the same modal- ity. The breast centre certification scheme focusses on real data, essential clinical skills, structure, and procedure.

EUSOMA has performed an analysis on the time trends of quality indicators in the EUSOMA-certified breast centres (EJC 85; 2017, van Dam et al. [17]) based on a total of 46,122 cases including DCIS, microinvasive and invasive breast cancer. In the study, EUSOMA has assessed the effect of certification analysing the evolution of QIs in the certified centres for the period 2006-2015. On average, the minimum standard was reached in all the mandatory QIs in the whole period analysed, the exception being the avoidance of repeat intervention for margins in DCIS. The target standard was reached for 2 QIs in 2006 and for 7 QIs in 2015.

Adherence to guidelines, reflected by a better performance of QIs, significantly improved over the years in the EUSOMA-certified centres. Constant monitoring of QIs allow these centres to strive to the highest level of care.

As reported in the review by the Joint Research Centre (JRC), in Europe there are 17 breast cancer-specific certification schemes available [18]. The JRC report analysis encompassed nine certification schemes, with three dedicated to screening certification overseen by the European Reference Organisation for Quality Assured Breast Screening and Diagnosis, the Mammography Co-operative in Germany, and the UK's National Health Service (NHS) National Breast Screening Programme, respectively. The report details national certification schemes run within European countries, including Germany, the $\mathrm{UK}$, and Switzerland.

The certification system for breast cancer centres overseen by the German Cancer Society (DKG) and German Society for Breast Disease (DGS) is part of a threestage model for oncological care according to the National Cancer Plan. Entities involved in the definitions of centre requirements include German Cancer Aid (DKH), DKG, the Association of the Scientific Medical Societies of Germany, the German Society of Obstetrics and Gynaecology, and 29 other professional associations. The certification process is run by Onkozert, the certification institute of the DKG. A questionnaire detailing breast centre requirements is used for internal self-assessment and is completed and reviewed before the site visit. Questionnaire items are based on existing guidelines (S3) and legal requirements. The scheme covers issues of screening, diagnosis, treatment, follow-up, rehabilitation, research, and training. A system of tumour documentation, containing patient data over at least 3 months, must be in place at the time of initial certification. Data are assessed and analysed every year. "Matrix of Quality" results are submitted for a formal, mandatory check, and on-site assessment is made by the auditor. Site visits run for 2 days, with a visiting party comprising of 3 auditors 
(oncology specialists trained in the auditing process). The visit encompasses the inspection of facilities and clinical areas, assessment of indicators, randomly sampled documents, revision of records, and interviewing of staff.

In the UK, the National Cancer Peer Review Programme administered by the National Cancer Action Team, is an all-cancer-sites project with a specific section dedicated to breast cancer. The project also involves the National Institute for Health and Care Excellence (NICE), the Care Quality Commission (CQC), and the NHS. The project uses the NICE Improving Outcome Guidance as an external reference. The scheme covers screening, diagnosis, treatment, rehabilitation, and research. A secure web-based database supports each stage of the peer review process. Each centre conducts an annual self-assessment and an internal validation of self-assessment every other year, utilising the measures contained within the Manual of Cancer Services [19]. At the completion of the external desktop review process, internal validation is either confirmed, confirmed with exceptions, or remains unconfirmed. The audit lasts 1 day both for the locality/ trust and for the network team. A peer-review visit is conducted by a multidisciplinary group consisting of clinicians, managers, and patients/carers. The visit agenda includes a preliminary review of evidence in preparation for the meeting, and a face-to-face meeting with the service. Following this, the review team assembles the final report. The programme does not deliver a certificate to the centre as it is a peer review.

Certification of breast centres under the "label de qualité" is run by the Swiss Anticancer League and the Swiss Senology Society. It provides a special rapid procedure of assessment of centres already certified by EUSOMA and/or DKG/DGS. The requirements are developed by consensus of a multidisciplinary working group on the basis of EUSOMA requirements. It also refers to the Swiss Radiology Society, the 2006 Parliamentary Resolution, and European Society of Medical Oncologists (ESMO) and St. Gallen recommendations. The scheme covers screening, diagnosis, treatment, follow-up, rehabilitation, research, and training. Yearly data transfer to the Swiss Senology Society database occurs for benchmarking. The breast centre is required to check compliance with the criteria before the assessment via a quality dashboard and questionnaire on structural requirements. The centre receives feedback from a decision board and can submit a request for an audit through a pre-defined questionnaire.

The audit takes 1 day and is run by a team of 3 auditors - among them at least 1 surgeon or 1 medical oncologist. The agenda includes a documental audit, visiting different services, interviews with healthcare professionals, and observing an MDM in process. At the completion of the audit, feedback is provided to the centre. During on-site visits, auditors review a graphical representation of the centre's organisational structure, personnel lists, clinical protocols, medical records, written information provided to patients regarding their rights and treatment, and patient complaints and feedback forms. Following the visit, the breast centre receives a formal written report [18].

The European Commission Initiative on Breast Cancer (ECIBC) manages the development of a European quality assurance scheme, defining a set of requirements and quality indicators for breast centres in Europe. These requirements are established on evidence-based guidelines. The scheme covers all services related to breast cancer care from screening to follow-up, and in some cases until end-of-life care. In the proposed European Breast QA scheme, classification of requirements will be proposed according to the following domains: clinical effectiveness, available facilities, resources and workforce, personal empowerment, and experience and safety. This scheme is anticipated to be launched as a voluntary certification design for Europe [20].

\section{Conclusion}

After nearly 20 years since the initial publication of EUSOMA guidelines regarding the requirements of a breast centre, it is hoped that the next step forward will be to ensure that in all European countries, breast cancer will be managed exclusively in breast centres, so that breast cancer patients do not have to travel or migrate to access the highest quality of care. This aim ultimately should be achieved through the joint efforts of health professionals, policy makers and patients with breast cancer and their advocates. Each breast centre needs to undergo an external audit based on defined requirements and quality indicators, to ensure to provide breast cancer patients the highest possible care and outcomes.

\section{Disclosure Statement}

The authors have no conflicts of interest to declare.

\section{Funding Sources}

No funding was received for the preparation of this paper.

\section{Author Contributions}

We acknowledge that all the authors listed above meet the four criteria for authorship as indicated by ICMJE recommendations. 


\section{References}

1 Cataliotti L, Costa A, Daly PA, Fallowfield L, Freilich G, Holmberg L, et al. Florence statement on breast cancer, 1998 forging the way ahead for more research on and better care in breast cancer. Eur J Cancer. 1999 Jan;35(1): 14-5.

2 Blamey RW, Cataliotti L, et al. The requirements of a specialist breast unit. Eur J Cancer. 2000 Dec;36(18):2288-93.

3 European Parliament Resolution on breast cancer in the European Union. Text adopted June 5, 2003. Strasbourg: European Parliament.

4 European Parliament Resolution on breast cancer in the enlarged European Union. Text adopted Oct 25, 2006. Strasbourg: European Parliament.

5 Declaration of the European Parliament of May 5, 2010 on the fight against breast cancer in the European Union. Brussels: European Parliament.

6 Cardoso F, Cataliotti L, Costa A, Knox S, Marotti L, Rutgers E, et al. European Breast Cancer Conference manifesto on breast centres/ units. Eur J Cancer. 2017;72, 244-50.

7 Wilson AR, Marotti L, Bianchi S, Biganzoli L, Claassen S, Decker T, et al.; EUSOMA (European Society of Breast Cancer Specialists). The requirements of a specialist Breast Centre. Eur J Cancer. 2013 Nov;49(17):3579-87.
8 Roohan PJ, Bickell NA, Baptiste MS, Therriault GD, Ferrara EP, Siu AL. Hospital volume differences and five-year survival from breast cancer. Am J Public Health. 1998 Mar;88(3): 454-7.

9 Greenup RA, Obeng-Gyasi S, Thomas S, Houck K, Lane WO, Blitzblau RC, et al. The Effect of Hospital Volume on Breast Cancer Mortality. Ann Surg. 2018 Feb;267(2):37581.

10 Kesson EM, Allardice GM, George WD, Burns HJ, Morrison DS. Effects of multidisciplinary team working on breast cancer survival: retrospective, comparative, interventional cohort study of 13,722 women. BMJ. 2012 Apr;344:e2718

11 Kung PT, Tsai WC. P0213: Effects of multidisciplinary care on survival of breast cancer: results from a national cohort study. Eur J Cancer. 2014;50(4):e69.

12 Foster TJ, Bouchard-Fortier A, Olivotto IA, Quan ML. Effect of Multidisciplinary Case Conferences on Physician Decision Making: Breast Diagnostic Rounds. Cureus. 2016 Nov; 8(11):e895.

13 Leff DR, Ho C, Thomas H, Daniels R, Side L, Lambert F, et al. A multidisciplinary team approach minimises prophylactic mastectomy rates. Eur J Surg Oncol. 2015 Aug;41(8): 1005-12.
14 Biganzoli L, Marotti L, Hart CD, Cataliotti L, Cutuli B, Kühn T, et al. Quality indicators in breast cancer care: an update from the EUSOMA working group. Eur J Cancer. 2017 Nov;86:59-81.

15 Breast Cancer Certification. www.breastcentrescertification.com

16 Kiderlen M, Ponti A, Tomatis M, Boelens PG, Bastiaannet E, Wilson R, et al.; eusomaDB Working Group. Variations in compliance to quality indicators by age for 41,871 breast cancer patients across Europe: a European Society of Breast Cancer Specialists database analysis. Eur J Cancer. 2015 Jul;51(10):1221-30.

17 van Dam PA, Tomatis M, Marotti L, Heil J, Mansel RE, Rosselli Del Turco M, et al. Time trends (2006-2015) of quality indicators in EUSOMA-certified breast centres. Eur J Cancer. 2017 Nov;85:15-22.

18 Deandrea S, Lerda D, Lopez Alcade J, Neamtiu L, Saz Parkinsons ZE, Uluturk A. Review and analysis of external quality assessment of breast cancer services in Europe. Brussels: Publication Office of the European Union; 2015. p. 112. Report No. EUR 27413OPOCE LF-NA 27413-EN-C (print), LF-NA-27413EN-N (online).

19 National Cancer Peer Review-National Cancer Action Team. Service user partnership group measures. London: NHS; April 7, 2011. p. 18, gateway ref. 15844 .

20 European Commission Initiative on Breast Cancer. https://ecibc.jrc.ec.europa.eu. 